Y. Kitaoka

Nagoya Math. J.

Vol. 70 (1978), 173-181

\title{
TENSOR PRODUCTS OF POSITIVE DEFINITE QUADRATIC FORMS III
}

\author{
YOSHIYUKI KITAOKA
}

In the previous papers [2], [3] we treated the following two questions. Let $L, M, N$ be positive definite quadratic lattices over $Z$ :

(i) If $L, M$ are indecomposable, then is $L \otimes M$ indecomposable?

(ii) Does $L \otimes M \cong L \otimes N$ imply $M \cong N$ ?

In this paper we discuss the uniqueness of decompositions with respect to tensor products. Our aim is to prove the following two theorems.

THEOREM 1. Let $L_{i}, M_{i}$ be indecomposable positive definite binary quadratic lattices with $L_{i}=\tilde{L}_{i}, M_{i}=\tilde{M}_{i}, m\left(L_{i}\right)=m\left(M_{i}\right)=1$. For any isometry $\sigma: \bigotimes_{i=1}^{n} L_{i} \cong \bigotimes_{i=1}^{n} M_{i}$, we have $\sigma=\bigotimes_{i=1}^{n} \sigma_{i}$ where $\sigma_{i}$ is an isometry from $L_{i}$ on $M_{i}$, changing the suffix if necessary.

THEOREM 2. Let $L_{i}, M_{i}$ be positive definite quadratic lattices with $\left[L_{i}: \tilde{L}_{i}\right]<\infty,\left[M_{i}: \tilde{M}_{i}\right]<\infty$. Assume that

(i ) $L_{i}$ (resp. $M_{i}$ ) is of E-type except at most one,

(ii) $s L_{i}=s M_{i}=Z$, and $m\left(L_{i}\right), m\left(M_{i}\right)$ are prime numbers, and

(iii) $\tilde{L}_{i}, \tilde{M}_{i}$ are indecomposable.

Then for any isometry $\sigma: \bigotimes_{i=1}^{n} L_{i} \cong \bigotimes_{i=1}^{m} M_{i}$ we have $n=m$ and $\sigma=\otimes \sigma_{i}$, where $\sigma_{i}$ is an isometry from $L_{i}$ on $M_{i}$, changing the suffix if necessary.

We must explain the notations and terminologies in two theorems. By a positive definite quadratic lattice we mean a lattice in a positive definite quadratic space over the rational number field $\boldsymbol{Q}$. For any quadratic space we use the same letter $Q, B$ which are the corresponding quadratic form and bilinear form $(2 B(x, y)=Q(x+y)-Q(x)-Q(y))$. Let $L$ be a positive definite quadratic lattice; then $s L$ denotes $\left\{\sum B\left(x_{i}, y_{i}\right)\right.$; $\left.x_{i}, y_{i} \in L\right\}$ and we put $m(L)=\min Q(x)$ where $x$ runs over non-zero elements of $L . \quad \mathfrak{M}(L)$ stands for $\{x \in L ; Q(x)=m(L)\}$, and $\tilde{L}$ is the sub-

Received June 21, 1977. 
module of $L$ spanned by elements of $\mathfrak{M}(L) . \quad L$ is called $E$-type if every element of $\mathfrak{M}(L \otimes M)$ is of the form $x \otimes y(x \in L, y \in M)$ for any positive definite quadratic lattice $M$. If either $s L \subseteq Z, m(L) \leq 6$ or $\operatorname{rank} L \leq 42$, then $L$ is of $E$-type [1].

§1. In this section we define a weighted graph and prove some properties.

DEFINITION. Let $A$ be a finite set, and [,] be a mapping from $A \times A$ into $\{t ; 0 \leq t \leq 1\}$ such that

(i) $\left[a, a^{\prime}\right]=1$ if and only if $a=\alpha^{\prime}$, and

(ii) $\left[a, \alpha^{\prime}\right]=\left[\alpha^{\prime}, a\right]$ for $a, a^{\prime}$ in $A$.

Then we call $(A,[]$,$) or simply A$ a weighted graph. A weighted graph $A$ is called connected if for any $x, y$ in $A$ there are elements $z_{i}$ of $A$ such that $x=z_{1}, y=z_{r}$ and $\left[z_{i}, z_{i+1}\right] \neq 0(i=1, \cdots, r-1)$. For weighted graphs $A, B$ we define the direct product $A \times B$ by $\left[(a, b),\left(a^{\prime}, b^{\prime}\right)\right]=$ $\left[a, a^{\prime}\right]\left[b, b^{\prime}\right]\left(a, a^{\prime} \in A, b, b^{\prime} \in B\right)$; then $A \times B$ is clearly a weighted graph. It is also clear that the direct product of connected weighted graphs is connected. $A$ bijection $f$ from $A$ on $B$ is called an isometry if $f$ satisfies $\left[f(\alpha), f\left(\alpha^{\prime}\right)\right]=\left[a, \alpha^{\prime}\right]$ for $a, \alpha^{\prime} \in A$.

LEMMA 1. Let $A, B, C$ be connected weighted graphs, and let $\sigma$ be an isometry from $A \times B$ on $A \times C$. If there are $b_{0} \in B, c_{0} \in C$ such that $\sigma\left(x, b_{0}\right)=\left(f(x), c_{0}\right)$ for every $x$ in $A$, then $f$ is an isometry from $A$ on $A$ and there is an isometry $g$ from $B$ on $C$ with $\sigma(x, y)=(f(x), g(y))$ $(x \in A, y \in B)$.

Proof. Since $\sigma$ is a bijection and $A$ is a finite set, $f$ is a bijection of $A$. Moreover for $a, a^{\prime}$ in $A$ we have $\left[a, a^{\prime}\right]=\left[\left(a, b_{0}\right),\left(a^{\prime}, b_{0}\right)\right]=$ $\left[\left(f(a), c_{0}\right),\left(f\left(\alpha^{\prime}\right), c_{0}\right)\right]=\left[f(a), f\left(a^{\prime}\right)\right]$. This means that $f$ is an isometry of A. Multiplying $f^{-1} \times \mathrm{id}_{C}$ to $\sigma$, we have only to prove the lemma in case of $f=1$. Put $S=\{\tilde{B} \subset B ; \sigma(a, b)=(a, c)$ for every $a \in A$ and $b \in \tilde{B}$, where $c$ is only dependent of $b\} . S$ is not empty since $S \ni\left\{b_{0}\right\}$. Take an element $B^{\prime}$ in $S$ such that $\# B^{\prime} \geq \# \tilde{B}$ for $\tilde{B}$ in $S$. If $B^{\prime}=B$, then we have $\sigma(a, b)=(a, g(b))$ for $a \in A, b \in B$. It is easy to see that $g$ is an isometry from $B$ on $C$, and this completes the proof. Now we assume $B^{\prime} \neq B$. We have to show that this implies a contradiction. Define a subset $C^{\prime}$ by $\sigma\left(A, B^{\prime}\right)=\left(A, C^{\prime}\right)$. Put $m=\max \left[b, b^{\prime}\right]$ where $b \in B^{\prime}, b^{\prime} \notin B^{\prime}$, and we may assume $m \geq \max \left[c, c^{\prime}\right]$ where $c \in C^{\prime}, c^{\prime} \notin C^{\prime}$, taking $\sigma^{-1}$ instead of $\sigma$ if necessary. Since $B$ is connected, $m$ is positive. 
Put $m=\left[b, b^{\prime}\right]\left(b \in B^{\prime}, b^{\prime} \notin B^{\prime}\right)$ and take any element $x$ of $A$. Put $\sigma\left(x, b^{\prime}\right)$ $=\left(x^{\prime}, c_{1}\right)$; then $c_{1}$ is not in $C^{\prime}$ since $c_{1} \in C^{\prime}$ implies $\left(x, b^{\prime}\right) \in \sigma^{-1}\left(A, C^{\prime}\right)=$ $\left(A, B^{\prime}\right)$. Putting $\sigma(x, b)=(x, c)$, we have $m=\left[b, b^{\prime}\right]=\left[(x, b),\left(x, b^{\prime}\right)\right]=$ $\left[(x, c),\left(x^{\prime}, c_{1}\right)\right]=\left[x, x^{\prime}\right]\left[c, c_{1}\right]$. If $x \neq x^{\prime}$, then $0<\left[x, x^{\prime}\right]<1$ implies a contradiction $m<\left[c, c_{1}\right] \leq m$. Hence $x^{\prime}=x$ follows. Thus we get $\sigma\left(x, b^{\prime}\right)=(x, c(x))(c(x) \in C)$ for every $x$ in $A$. For $x, y$ in $A$ with $[x, y]$ $\neq 0,[x, y]=\left[\left(x, b^{\prime}\right),\left(y, b^{\prime}\right)\right]=[(x, c(x)),(y, c(y))]=[x, y][c(x), c(y)]$ implies $[c(x), c(y)]=1$, and so $c(x)=c(y)$. Since $A$ is connected, this yields that $c(x)$ in $C$ is independent of $x$ in $A$, and then it implies a contradiction $B^{\prime} \cup\left\{b^{\prime}\right\} \in S$ and $\#\left(B^{\prime} \cup\left\{b^{\prime}\right\}\right)>\# B^{\prime}$.

LeMma 2. Let $L$ be a positive definite quadratic lattice. For $x, y$ in $L$ we put $[x, y]=|B(x, y)| / m(L)$. Then $(\mathfrak{M}(L) / \pm 1,[]$,$) is a weighted$ graph and it is connected if and only if $\tilde{L}$ is indecomposable.

Proof. Take $x, y$ in $\mathfrak{M}(L)$; then $x= \pm y$ if and only if $|B(x, y)|=$ $m(L)$. Moreover $B(x, y)^{2} \leq Q(x) Q(y)=m(L)^{2}$ implies that $\mathfrak{M}(L) / \pm 1$ is a weighted graph. The latter part is obvious.

We say that $(\mathfrak{M}(L) / \pm 1,[]$,$) is a weighted graph associated to L$.

§2. Let $L_{i}, M_{j}$ be positive definite quadratic lattices and let $\sigma$ be an isometry from $\bigotimes_{i=1}^{n} L_{i}$ on $\otimes_{j=1}^{m} M_{j}$. Suppose that

( i ) $\mathfrak{M}\left(\otimes L_{i}\right)=\otimes \mathfrak{M}\left(L_{i}\right), \mathfrak{M}\left(\otimes M_{j}\right)=\otimes \mathfrak{M}\left(M_{j}\right)$,

(ii) $\left[L_{i}: \tilde{L}_{i}\right],\left[M_{j}: \tilde{M}_{j}\right]<\infty$ for every $i, j$,

(iii) $\mathfrak{M}\left(L_{i}\right) / \pm 1, \mathfrak{M}\left(M_{j}\right) / \pm 1$ are connected weighted graphs for every $i, j$.

Let $A, B, A_{i}, B_{i}$ be weighted graphs associated to $\otimes L_{i}, \otimes M_{i}, L_{i}, M_{i}$ respectively. Then $\sigma$ induces an isometry from $A=\prod_{i=1}^{n} A_{i}$ on $B=\prod_{i=1}^{m} B_{i}$ which is denoted by the same letter $\sigma$.

THEOREM. If it follows that $n=m, \sigma=\prod_{i=1}^{n} \sigma_{i}$ where $\sigma_{i}$ is an isometry from $A_{i}$ on $B_{i}$, changing the suffix if necessary, then we have $\sigma=\bigotimes_{i=1}^{n} \mu_{i}$ where $\mu_{i}$ is an isometry from $L_{i}$ on $M_{i}$, changing the suffix if necessary.

Proof. We may assume $\sigma=\prod \sigma_{i}$ where $\sigma_{i}$ is an isometry from $A_{i}$ on $B_{i}$. By the same letter $\sigma_{i}$ we denote a mapping from $\mathfrak{M}\left(L_{i}\right)$ on $\mathfrak{M}\left(M_{i}\right)$ which induces an isometry $\sigma_{i}$ from $A_{i}=\mathfrak{M}\left(L_{i}\right) / \pm 1$ on $B_{i}=\mathfrak{M}\left(M_{i}\right) / \pm 1$. Fix any element $e_{i}$ in $\mathfrak{M}\left(L_{i}\right)(i \geq 2)$. Then $\sigma\left(e \otimes e_{2} \otimes \cdots \otimes e_{n}\right)= \pm \sigma_{1}(e)$ 
$\otimes \sigma_{2}\left(e_{2}\right) \otimes \cdots \otimes \sigma_{n}\left(e_{n}\right)$ holds for every $e$ in $\mathfrak{M}\left(L_{1}\right)$. Putting $\pm \sigma_{1}(e)=\mu_{1}(e)$, then $\sigma\left(e \otimes e_{2} \otimes \cdots \otimes e_{n}\right)=\mu_{1}(e) \otimes \sigma_{2}\left(e_{2}\right) \otimes \cdots \otimes \sigma_{n}\left(e_{n}\right)$ for any $e$ in $\mathfrak{M}\left(L_{1}\right)$. This means that $\mu_{1}$ is an isometry from $\tilde{L}_{1}$ onto $\tilde{M}_{1}$. Since $M_{1} \otimes \sigma_{2}\left(e_{2}\right)$ $\otimes \cdots \otimes \sigma_{n}\left(e_{n}\right)$ is a direct summand of $\otimes M_{i}$ and $\left[L_{1}: \tilde{L}_{1}\right]<\infty, \mu_{1}$ is an isometry from $L_{1}$ into $M_{1}$. Similarly we get an isometry $\mu_{i}$ from $L_{i}$ into $M_{i}$ so that $\sigma\left(e_{1} \otimes \cdots \otimes e_{n}\right)= \pm \mu_{1}\left(e_{1}\right) \otimes \cdots \otimes \mu_{n}\left(e_{n}\right)$ for $e_{i}$ in $\mathfrak{M}\left(L_{i}\right)$, where \pm may depend on the choice of $e_{i}$. Since $\mathfrak{M}\left(L_{i}\right) / \pm 1$ is connected and moreover $\delta=\delta^{\prime}$ if $\sigma\left(e_{1} \otimes \cdots \otimes e_{n}\right)=\delta \mu_{1}\left(e_{1}\right) \otimes \cdots \otimes \mu_{n}\left(e_{n}\right), \sigma\left(e_{1}^{\prime} \otimes \cdots\right.$ $\left.\otimes e_{n}^{\prime}\right)=\delta^{\prime} \mu_{1}\left(e_{1}^{\prime}\right) \otimes \cdots \otimes \mu_{n}\left(e_{n}^{\prime}\right)$ are not orthogonal, \pm does not depend on the choice of $e_{i}$. Thus we get $\sigma=\otimes \mu_{i}$, taking $-\mu_{1}$ if necessary. Since $\sigma$ is an onto-mapping, $\mu_{i}$ is an isometry from $L_{i}$ on $M_{i}$. This completes the proof.

§3. First we discuss the case of Theorem 1 . Let $L$ be an indecomposable binary positive definite quadratic lattice with $L=\tilde{L}, m(L)$ $=1$. Then $L$ has a basis $\left\{e_{1}, e_{2}\right\}$ so that $Q\left(e_{1}\right)=Q\left(e_{2}\right)=1,0<B\left(e_{1}, e_{2}\right)$ $\leq \frac{1}{2}$, and moreover we have $\mathfrak{M}(L)=\left\{ \pm e_{1}, \pm e_{2}, \pm\left(e_{1}-e_{2}\right)\right\} \quad\left( \pm\left(e_{1}-e_{2}\right)\right.$ happens only when $\left.B\left(e_{1}, e_{2}\right)=\frac{1}{2}\right)$. Let $A_{L}$ be a weighted graph associated to $L$; then $A_{L}$ is connected. \# $A_{L}$ is two for $B\left(e_{1}, e_{2}\right)<\frac{1}{2}$. If $B\left(e_{1}, e_{2}\right)$ $=\frac{1}{2}$, then $\# A_{L}=3$ and $\left[a_{i}, a_{j}\right]=\frac{1}{2}$ for $i \neq j$ where we put $A_{L}=\left\{a_{1}, a_{2}, a_{3}\right\}$.

Let $L_{i}, M_{i}, \sigma$ be as in Theorem 1 ; then $L_{i}, M_{i}$ are of $E$-type, and define $A, A_{i}, B, B_{i}$ and $\sigma$ as in $\S 2$; then we have

LEMMA 3. $\sigma=\prod \sigma_{i}$ where $\sigma_{i}$ is an isometry from $A_{i}$ on $B_{i}$, changing the suffix if necessary.

Proof. We prove this by the induction with respect to \#A. Put $m=\max \left[a, a^{\prime}\right]=\max \left[b, b^{\prime}\right]$ where $a, a^{\prime} \in A, a \neq a^{\prime}$ and $b, b^{\prime} \in B, b \neq b^{\prime}$. Since $A_{i}, B_{i}$ are indecomposable, we get $0<m \leq \frac{1}{2}$. Take $a \neq a^{\prime}$ in $A$ with $\left[a, a^{\prime}\right]=m$. Putting $a=\prod a_{i}, a^{\prime}=\prod a_{i}^{\prime}, m=\prod\left[a_{i}, a_{i}^{\prime}\right]$ follows. Noting $\left[a_{i}, a_{i}^{\prime}\right]<1$ for $a_{i} \neq a_{i}^{\prime}$, the maximality of $m$ implies that there is an index $j$ such that $\left[a_{i}, a_{i}^{\prime}\right]=1$, i.e., $a_{i}=a_{i}^{\prime}$ for $i \neq j$, and $a_{j} \neq a_{j}^{\prime}$. We may assume $j=1$, and similarly $\sigma(a)=\prod b_{i}, \sigma\left(a^{\prime}\right)=\prod b_{i}^{\prime}, b_{i}=b_{i}^{\prime}$ for $i>1$ and $b_{1} \neq b_{1}^{\prime}$. Then $m=\left[a_{1}, a_{1}^{\prime}\right]=\left[b_{1}, b_{1}^{\prime}\right]$ follows. If $m<\frac{1}{2}$, then $A_{1}=\left\{a_{1}, a_{1}^{\prime}\right\}, B_{1}=\left\{b_{1}, b_{1}^{\prime}\right\}$ and $\sigma\left(A_{1} \times \prod_{i=2}^{n} a_{i}\right)=B_{1} \times \prod_{i=2}^{n} b_{i}$. Hence Lemma 1 and the assumption of the induction completes the proof. Suppose $m=\frac{1}{2}$; then there is an element $a_{1}^{\prime \prime}$ in $A_{1}$ so that $A_{1}=\left\{a_{1}, a_{1}^{\prime}, a_{1}^{\prime \prime}\right\}$ and $\left[a_{1}, a_{1}^{\prime \prime}\right]=\left[a_{1}^{\prime}, a_{1}^{\prime \prime}\right]=\frac{1}{2}$. Put $\sigma\left(a_{1}^{\prime \prime} \times \prod_{i=2}^{n} a_{i}\right)=\prod b_{i}^{\prime \prime} ;$ then $\left[a_{1}, a_{1}^{\prime \prime}\right]=$ 
$\left[a_{1}^{\prime}, a_{1}^{\prime \prime}\right]=\frac{1}{2}$ implies $\left[b_{1}, b_{1}^{\prime \prime}\right] \prod_{i=2}^{n}\left[b_{i}^{\prime \prime}, b_{i}\right]=\left[b_{1}^{\prime}, b_{1}^{\prime \prime}\right] \prod_{i=2}^{n}\left[b_{i}^{\prime \prime}, b_{i}\right]=\frac{1}{2}$. Suppose $b_{1}=b_{1}^{\prime \prime}$; then $\prod_{i=2}^{n}\left[b_{i}^{\prime \prime}, b_{i}\right]=\frac{1}{2}$, and so $\left[b_{1}^{\prime}, b_{1}^{\prime \prime}\right]=1$, that is, $b_{1}^{\prime}=b_{1}^{\prime \prime}=b_{1}$. This is a contradiction. Hence we have $b_{1} \neq b_{1}^{\prime \prime}$, and then $\left[b_{1}, b_{1}^{\prime \prime}\right]=\frac{1}{2}$. Therefore $b_{i}^{\prime \prime}=b_{i}$ for $i \geq 2$ and $\sigma\left(A_{1} \times \prod_{i=2}^{n} a_{i}\right)=B_{1} \times \prod_{i=2}^{n} b_{i}$. This completes the proof as above.

Now Theorem 1 follows from Theorem in $\S 2$.

Next we discuss the case of Theorem 2.

LemMA 4. Let $a_{i}, b_{i} \in Z$ and $0<b_{i}<a_{i}$, and let $a_{i}$ be prime. Put $\prod_{i=1}^{n}\left(b_{i} / a_{i}\right)=b / a,(a, b)=1$. Then $a>a_{i}$ for some $i$ if $n \geq 2$.

Proof. We may suppose $a_{1} \leq \cdots \leq a_{n}$, and assume $a \leq a_{i}$ for any $i$. Since a divides $\prod a_{i}$, we have $a=a_{1} . \quad b_{1} \prod_{i=2}^{n}\left(b_{i} / a_{i}\right)=b$ and $a_{i} \nmid b_{1}$ imply $\prod_{i=2}^{n} a_{i} \mid \prod_{i=2}^{n} b_{i}$. This contradicts $0<b_{i}<a_{i}$.

LEMMA 5. Let $A_{i}, B_{i}$ be connected weighted graphs with \# $A_{i}>1$, $\# B_{i}>1$, and let $p_{i}, q_{i}$ be primes. Suppose

$$
\left\{[x, y] ; x, y \in A_{i}\right\} \subset\left\{a / p_{i} ; a=0,1, \cdots, p_{i}\right\}
$$

and

$$
\left\{[x, y] ; x, y \in B_{i}\right\} \subset\left\{b / q_{i} ; b=0,1, \cdots, q_{i}\right\} .
$$

If $\sigma$ is an isometry from $\prod_{i=1}^{n} A_{i}$ on $\prod_{i=1}^{m} B_{i}$, then $n=m$ and $\sigma=\prod \sigma_{i}$ where $\sigma_{i}$ is an isometry from $A_{i}$ on $B_{i}$, changing the suffix if necessary.

Proof. We prove by the induction with respect to \# $\prod_{i=1}^{n} A_{i}$. Since $A_{i}$ is connected and $\# A_{i}>1$, for any element $a$ in $A_{i}$ there is an element $a^{\prime}$ in $A_{i}$ such that $0<\left[a, a^{\prime}\right]<1$. If $\left[a, a^{\prime}\right] \neq 0,1$ for $a, a^{\prime}$ in $A_{i}$, then the denominator of $\left[a, a^{\prime}\right]$ is a prime $p_{i}$. Without loss of generality we may assume $p_{1}=\cdots=p_{k}<p_{k+1} \leq \cdots \leq p_{n}, q_{1}=\cdots=q_{h}$ $<q_{h+1} \leq \cdots \leq q_{m}$. Put $A=\prod_{i=1}^{n} A_{i}, B=\prod_{i=1}^{m} B_{i}$, and fix any element $a=\prod a_{i}$ of $A$. Suppose that the minimal value of the denominator of $\left[a, a^{\prime}\right]$ with $\left[a, \alpha^{\prime}\right] \neq 0,1 \quad\left(a^{\prime} \in A\right)$ is taken by $a^{\prime}=\prod a_{i}^{\prime} \in A$. Then the above remark and Lemma 4 imply $a_{i}^{\prime}=a_{i}$ for $i \neq j$, and $a_{j}^{\prime} \neq a_{j}$ for some $j$ and so the minimal value is obviously $p_{1}$, and $j \leq k$. On the other hand, by virtue of Lemma 4 and the connectedness of $A_{i}$, it is easy to see that $A_{1} \times \cdots \times A_{k} \times a_{k+1} \times \cdots \times a_{n}$ is a subset of $A$ consisting of elements $z$ such that there are elements $z_{1}=a, \cdots, z_{r}=z$ of $A$ satisfying that the denominator of $\left[z_{i}, z_{i+1}\right]$ is $p_{1}$ for $i=1, \cdots, r-1$. From the similar argument for $\sigma(a)=\prod b_{i}$ in $B$ follows that the 
corresponding minimal denominator is $q_{1}$, and the corresponding subset of $B$ for $q_{1}, \sigma(a)$ instead of $p_{1}, a$ is $B_{1} \times \cdots \times B_{h} \times b_{h+1} \times \cdots \times b_{m}$. Since $\sigma$ is an isometry, we have $p_{1}=q_{1}$, and so $\sigma\left(A_{1} \times \cdots \times A_{k} \times a_{k+1}\right.$ $\left.\times \cdots \times a_{n}\right)=B_{1} \times \cdots \times B_{h} \times b_{h+1} \times \cdots \times b_{m}$ by their definitions. This implies that $A_{1} \times \cdots \times A_{k}$ and $B_{1} \times \cdots \times B_{h}$ are isometric. Therefore Lemma 1 and the assumption of the induction completes the proof if $n>k$. Thus we may suppose $n=k$. Then $\sigma(A)=\prod_{i=1}^{h} B_{i} \times b_{h+1} \times$ $\cdots \times b_{m}$ implies $h=m$. Moreover we have $n=m$ since the maximal value of the denominators of $\left[a, a^{\prime}\right]\left(a, a^{\prime} \in A\right)$ (resp. $\left.\left[b, b^{\prime}\right]\left(b, b^{\prime} \in B\right)\right)$ is $p_{1}^{n}\left(\right.$ resp. $\left.p_{1}^{m}\right)$, and they are equal. For simplicity we put $p_{1}=p$ in the following.

(i) Assume that $A_{1}$ contains distinct three elements $x_{1}, x_{2}, x_{3}$ such $\left[x_{1}, x_{2}\right]\left[x_{2}, x_{3}\right]\left[x_{3}, x_{1}\right] \neq 0$. Fix any element $a_{i}$ in $A_{i}(i \geq 2)$, and put $\sigma\left(x_{k} \prod_{i=2}^{n} a_{i}\right)=\prod_{j=1}^{n} b_{k, j}\left(b_{k, j} \in B_{j}\right)$; then $\left[x_{i}, x_{k}\right]=\prod_{j=1}^{n}\left[b_{i, j}, b_{k, j}\right] \neq 0$. Since $0<\left[b_{i, j}, b_{k, j}\right] \leq 1$ and the denominator of $\left[b_{i, j}, b_{k, j}\right]$ is $p$ if $b_{i, j} \neq b_{k, j}$, compairing the denominators of both sides, we have $b_{i, j}=b_{k, j}$ for any $j$ except one index if $i \neq k$. Without loss of generality we may assume $b_{1,1} \neq b_{2,1}, \quad b_{1, i}=b_{2, i} \quad(i \geq 2)$. Similarly we may assume $b_{2, k}=b_{3, k}$ for $k \neq t$. If $t \geq 2$, then $b_{1, j}=b_{2, j}=b_{3, j}$ for $j \neq 1$, $t$. This implies $\left[x_{1}, x_{3}\right]$ $=\left[b_{1,1}, b_{3,1}\right]\left[b_{1, t}, b_{3, t}\right]=\left[b_{1,1}, b_{2,1}\right]\left[b_{2, t}, b_{3, t}\right]$. The denominator of the left (resp. right) side is $p$ (resp. $p^{2}$ ) since $b_{1,1} \neq b_{2,1}, b_{2, t} \neq b_{3, t}$. This is a contradiction. Hence we get $t=1$, and so $b_{2,1} \neq b_{3,1}, b_{2, j}=b_{3, j}(j \geq 2)$. Thus we may put $\sigma\left(x_{k} \times \prod_{i=2}^{n} a_{i}\right)=y_{k} \times \prod_{i=2}^{n} b_{i}\left(y_{k} \in B_{1}, b_{i} \in B_{i}\right)$. Take an element $a_{n}^{\prime}$ in $A_{n}$ such that $\left[a_{n}, a_{n}^{\prime}\right] \neq 0,1$. Similarly we get $\sigma\left(x_{k} \times\right.$ $\left.\prod_{i=2}^{n-1} a_{i} \times a_{n}^{\prime}\right)=z_{k} \times \prod_{i \neq j} b_{i}^{\prime}$ for some $z_{k}$ in $B_{j}$ and $b_{i}^{\prime}$ in $B_{i}$. Suppose $j \neq 1$, then $\left[a_{n}, a_{n}^{\prime}\right]=\left[x_{k} \times \prod_{i=2}^{n} a_{i}, x_{k} \times \prod_{i=2}^{n-1} a_{i} \times a_{n}^{\prime}\right]=\left[y_{k}, b_{1}^{\prime}\right]\left[b_{j}, z_{k}\right] \times$ $\prod_{i \neq 1, j}\left[b_{i}, b_{i}^{\prime}\right]$. We note that the denominator of the left side is $p$. If $b_{i} \neq b_{i}^{\prime}$ for $i \neq 1, j$, then $\left[y_{k}, b_{1}^{\prime}\right]=1$, and so $y_{1}=y_{2}=y_{3}$. This implies a contradiction $x_{1}=x_{2}=x_{3}$. Hence $b_{i}=b_{i}^{\prime}$ for $i \neq 1, j . \quad b_{1}^{\prime} \neq y_{1}$ implies $b_{j}=z_{1}\left(\neq z_{2}, z_{3}\right)$, and so we get $y_{2}=y_{3}=b_{1}^{\prime}$, taking $k=2$ or 3 . This is a contradiction. Hence we have $b_{1}^{\prime}=y_{1}$, and similarly $b_{1}^{\prime}=y_{2}$. This contradicts $x_{1} \neq x_{2}$. Hence $j$ equals 1 , and we may put $\sigma\left(x_{k} \times \prod_{i=2}^{n-1} a_{i}\right.$ $\left.\times a_{n}^{\prime}\right)=z_{k} \times \prod_{i=2}^{n} b_{i}^{\prime}\left(z_{k} \in B_{1}, b_{i}^{\prime} \in B_{i}\right) . \quad \sigma\left(x_{k} \times \prod_{i=2}^{n} a_{i}\right)=y_{k} \times \prod_{i=2}^{n} b_{i}$ implies $\left[x_{k}, x_{h}\right]\left[a_{n}, a_{n}^{\prime}\right]=\left[y_{k}, z_{h}\right] \prod_{i=2}^{n}\left[b_{i}, b_{i}^{\prime}\right]$. Putting $k=h$, and compairing the denominators we have $b_{i}=b_{i}^{\prime}$ for any $i \geq 2$ except at most one $i$. Putting $k \neq h$, the denominator of the left hand equals $p^{2}$. Hence the exceptional suffix exists. Then putting $k=h$ again, we have $y_{k}=z_{k}$ 
for $k=1,2,3$. Thus we have $\sigma\left(x_{k} \times \prod_{i=2}^{n-1} a_{i} \times a_{n}^{\prime}\right)=y_{k} \times \prod_{i=2}^{n} b_{i}^{\prime}$. Doing the similar operations for $a_{i}, a_{n}^{\prime}$, we have $\sigma\left(x_{k} \times \prod_{i=2}^{n} A_{i}\right) \subset y_{k} \times \prod_{i=2}^{n} B_{i}$ since $A_{i}$ is connected. Similarly $\sigma^{-1}\left(y_{k} \prod_{i=2}^{n} b_{i}\right)=x_{k} \prod_{i=2}^{n} a_{i}$ and $\left[y_{1}, y_{2}\right]$ $\times\left[y_{2}, y_{3}\right]\left[y_{3}, y_{1}\right] \neq 0$ imply $\sigma^{-1}\left(y_{k} \prod_{i=2}^{n} B_{i}\right) \subset x_{k} \times \prod_{i=2}^{n} A_{i}$, and so $\sigma\left(x_{k} \times\right.$ $\left.\prod_{i=2}^{n} A_{i}\right)=y_{k} \times \prod_{i=2}^{n} B_{i}$. This implies $\prod_{i=2}^{n} A_{i} \cong \prod_{i=2}^{n} B_{i}$, and then Lemma 1 and the assumption of the induction completes the proof.

(ii) Suppose that $A_{1}$ contains distinct four elements $x_{i}$ such that $\left[x_{1}, x_{2}\right]\left[x_{1}, x_{3}\right]\left[x_{1}, x_{4}\right] \neq 0,\left[x_{2}, x_{3}\right]=\left[x_{2}, x_{4}\right]=\left[x_{3}, x_{4}\right]=0$. Fix any element $a_{i}$ in $A_{i}(i \geq 2)$. Put $\sigma\left(x_{k} \times \prod_{i=2}^{n} a_{i}\right)=\prod_{i=1}^{n} b_{k, i}$; then $\left[x_{k}, x_{1}\right]=\prod_{i=1}^{n}\left[b_{k, i}, b_{1, i}\right]$ $\neq 0$. Since the denominator of the left hand is $p$ for $k \neq 1$, there is a number $t_{k}$ such that $b_{k, i}=b_{1, i}$ for $i \neq t_{k}$, and $b_{k, t_{k}} \neq b_{1, t_{k}}$.

a) Suppose that $t_{2}, t_{3}, t_{4}$ are distinct.

$\left[x_{3}, x_{4}\right]=0$ implies $\left[b_{3, i}, b_{4, i}\right]=0$ for some $i$. Since $b_{k, j}=b_{1, j}$ for $j \neq$ $t_{2}, t_{3}, t_{4}, i$ equals $t_{2}, t_{3}$ or $t_{4}$. If $i=t_{2}$, then $b_{3, t_{2}}=b_{1, t_{2}}=b_{4, t_{2}}$ implies a contradiction $\left[b_{3, t_{2}}, b_{4, t_{2}}\right]=1$. Similarly $i=t_{3}$ or $i=t_{4}$ implies a contradiction.

b) Suppose that $t_{2}=t_{3} \neq t_{4}$.

$\left[x_{3}, x_{4}\right]=0$ implies $\left[b_{3, i}, b_{4, i}\right]=0$ for some $i . b_{k, j}=b_{1, j}$ for $j \neq t_{k}$ yields $i=t_{2}$ or $t_{4}$. $i=t_{2}$ implies $b_{4, t_{2}}=b_{1, t_{2}}$, and so $\left[b_{3, i}, b_{1, i}\right]=0$. This contradicts $\left[x_{3}, x_{1}\right] \neq 0$. Similarly $i=t_{4}$ is a contradiction.

Similarly $t_{2} \neq t_{3}=t_{4}$ or $t_{2}=t_{4} \neq t_{3}$ implies a contradiction. Hence we have $t_{2}=t_{3}=t_{4}=1$ (say). Thus we may assume $\sigma\left(x_{k} \times \prod_{i=2}^{n} a_{i}\right)=$ $y_{k} \times \prod_{i=2}^{n} b_{i}\left(y_{k} \in B_{1}, \quad b_{i} \in B_{i}\right)$. Take an element $a_{n}^{\prime}$ in $A_{n}$ with $\left[a_{n}, a_{n}^{\prime}\right]$ $\neq 0,1$, and put $\sigma\left(x_{k} \times \prod_{i=2}^{n-1} a_{i} \times a_{n}^{\prime}\right)=z_{k} \prod_{i \neq j} b_{i}^{\prime} \quad\left(z_{k} \in B_{j}, b_{i}^{\prime} \in B_{i}\right)$. Assume $j \neq 1$; then $\left[x_{k} \times \prod_{i=2}^{n} a_{i}, x_{t} \times \prod_{i=2}^{n-1} a_{i} \times a_{n}^{\prime}\right]=\left[x_{k}, x_{t}\right] \times\left[a_{n}, a_{n}^{\prime}\right]$ $=\left[y_{k}, b_{1}^{\prime}\right]\left[b_{j}, z_{t}\right] \prod_{i \neq 1, j}\left[b_{i}, b_{i}^{\prime}\right] . \quad\left[x_{1}, x_{t}\right]\left[a_{n}, a_{n}^{\prime}\right] \neq 0$ implies $\left[b_{j}, z_{t}\right] \neq 0 \quad(t=$ $1,2,3,4),\left[b_{i}, b_{i}^{\prime}\right] \neq 0$ for $i \neq 1, j$. Similarly $\left[x_{k}, x_{1}\right] \neq 0$ implies $\left[y_{k}, b_{1}^{\prime}\right]$ $\neq 0(k=1,2,3,4)$. This means $\left[x_{k}, x_{t}\right]\left[a_{n}, a_{n}^{\prime}\right] \neq 0$ for any $k, t$ and contradicts $\left[x_{2}, x_{3}\right]=0$. Thus we have $j=1$, and $\left[x_{k}, x_{t}\right] . \quad\left[a_{n}, a_{n}^{\prime}\right]=\left[y_{k}, z_{t}\right]$ $\times \prod_{i=2}^{n}\left[b_{i}, b_{i}^{\prime}\right]$. Since the denominator of the left hand for $k=1, t=2$ is $p^{2}$, there is at least one suffix $i$ such that $b_{i} \neq b_{i}^{\prime}$. Moreover the denominator of the left side for $k=t$ is $p$. Hence there is no such suffix except $i$, and this yields $\left[y_{k}, z_{k}\right]=1$, i.e., $y_{k}=z_{k}$. As the proof of the case (i) we have $\sigma\left(x_{k} \times \prod_{i=2}^{n} A_{i}\right)=y_{k} \times \prod_{i=2}^{n} B_{i}$ and complete the proof for the case (ii) by the induction and Lemma 1.

For a weighted graph $W$ we make a usual graph, joining two ele- 
ments $x, y$ with $[x, y] \neq 0$. Then, by virtue of (i), (ii), we may assume that $A_{\imath}, B_{i}$ do not contain subgraphs

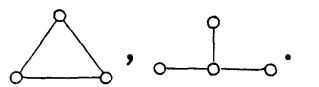
Hence $A_{i}, B_{i}$ are $\multimap \cdots$ or $\rightarrow$ as graphs.

(iii) Suppose that $A_{1}$ contains three distinct elements $x_{1}, x_{2}, x_{3}$ such that $\left[x_{1}, x_{2}\right] \neq 0,\left[x_{2}, x_{3}\right] \neq 0,\left[x_{1}, x_{3}\right]=0$, i.e., $\begin{array}{ccc}x_{1} & x_{2} & x_{3} \\ 0 & 0\end{array}$. Take any element $a_{i}$ in $A_{i}$, and put $\sigma\left(x_{k} \prod_{i=2}^{n} a_{i}\right)=\prod_{i=1}^{n} b_{k, i}\left(b_{k, i} \in B_{i}\right)$. Compairing the denominators of $\left[x_{k}, x_{t}\right]=\prod_{i=1}^{n}\left[b_{k, i}, b_{t, i}\right]$, there are numbers $q$, so that $b_{1, i}=b_{2, i}$ for $i \neq q, b_{2, i}=b_{3, i}$ for $i \neq s . \quad q \neq s$ implies $b_{1, i}=b_{2, i}=b_{3, i}$ for $i \neq q, s, \quad b_{2, q}=b_{3, q}$ and $b_{1, s}=b_{2, s}$, and then we have $\left[x_{1}, x_{3}\right]=$ $\prod_{i=1}^{n}\left[b_{1, i}, b_{3, i}\right]=\left[b_{1, q}, b_{3, q}\right]\left[b_{1, s}, b_{3, s}\right]=\left[b_{1, q}, b_{2, q}\right]\left[b_{2, s}, b_{3, s}\right]=0$. This contra$\operatorname{dicts}\left[x_{1}, x_{2}\right]\left[x_{2}, x_{3}\right] \neq 0$. Thus we may assume $q=s=1$ (say), and $\sigma\left(x_{k} \prod_{i=2}^{n} a_{i}\right)=y_{k} \prod_{i=2}^{n} b_{i}\left(y_{k} \in B_{1}, \quad b_{i} \in B_{i}\right)$. Doing the similar thing for \begin{tabular}{ccc}
$x_{2}$ & $x_{3}$ & $x_{4}$ \\
\hdashline & $0^{\prime}$
\end{tabular} , we have $\sigma\left(x_{k} \times \prod_{i=2}^{n} a_{i}\right)=z_{k} \prod_{i \neq j} b_{i}^{\prime} \quad\left(z_{k} \in B_{j}, \quad b_{i}^{\prime} \in B_{i}\right)$ for $k=2,3,4$. Compairing the case $k=2,3$, we get $z_{2}=b_{j}=z_{3}$ if $j \neq 1$. This is a contradiction, and so $j=1$. This means $b_{i}^{\prime}=b_{i}$ for $i \geq 2$ and $\sigma\left(x_{4} \prod_{i=2}^{n} a_{i}\right)=z_{4} \prod_{i=2}^{n} b_{i}$. Since $A_{1}$ is $-\ldots \multimap$ or $\sigma\left(x \times \prod_{i=2}^{n} a_{i}\right)=f(x) \times \prod_{i=2}^{n} b_{i}$ for any $x$ in $A_{1}$, that is, $\sigma\left(A_{1} \times \prod_{i=2}^{n} a_{i}\right)$ $\subset B_{1} \times \prod_{i=2}^{n} b_{i}$. Similarly we have $\sigma^{-1}\left(B_{1} \times \prod_{i=2}^{n} b_{i}\right) \subset A_{1} \times \prod_{i=2}^{n} a_{i}$ and so $\sigma\left(A_{1} \times \prod_{i=2}^{n} a_{i}\right)=B_{1} \times \prod_{i=2}^{n} b_{i}$. Lemma 1 and the induction complete the proof.

(iv) By virtue of (i), (ii), (iii) we have only to prove the case that $\# A_{i}=\# B_{i}=2$. Put $m=\max \left[a, a^{\prime}\right]\left(a, a^{\prime} \in A, a \neq a^{\prime}\right)$ and assume $m=$ $\left[a, a^{\prime}\right]$ for $a=\prod_{i=1}^{n} a_{i}, a^{\prime}=\prod_{i=1}^{n} a_{i}^{\prime}$. Since $\left[a_{i}, a_{i}^{\prime}\right]<1$ if $a_{i} \neq a_{i}^{\prime}$, by the definition, there is a suffix $t$ so that $a_{i}=a_{i}^{\prime}$ for $i \neq t$ and $a_{t} \neq a_{t}^{\prime}$. Putting $\sigma(a)=\prod b_{i}, \sigma\left(a^{\prime}\right)=\prod b_{i}^{\prime}$, there is a suffix $s$ so that $b_{i}=b_{i}^{\prime}$ for $i \neq s$, and $b_{s} \neq b_{s}^{\prime}$. Without loss of generality we may assume $t=s=1$; then $A_{1}=\left\{a_{1}, a_{1}^{\prime}\right\}, B_{1}=\left\{b_{1}, b_{1}^{\prime}\right\}$ and $\left[a_{1}, a_{1}^{\prime}\right]=\left[b_{1}, b_{1}^{\prime}\right]=m$. Hence $A_{1} \cong B_{1}$ and $\sigma\left(A_{1} \times \prod_{i=2}^{n} a_{i}\right)=B_{1} \times \prod_{i=2}^{n} b_{i}$. Lemma 1 and the assumption of the induction complete the proof of Lemma 4 .

To complete the proof of Theorem 2 we need only to prove that the cardinalities of weighted graphs associated to $L_{i}, M_{i}$ are not 1 . It follows immediately from the assumption (ii).

Let $L$ be an indecomposable positive definite quadratic lattice, and 
put $A=\mathfrak{M}(L) / \pm 1$ and we consider $A$ as a weighted graph by $[x, y]=$ $|B(x, y)| / m(L)$ for $x, y \in \mathfrak{M}(L) / \pm 1$ as above. We call such a weighted graph a quadratic weighted graph associated to $L$. Then the following questions arise.

(i) Let $A_{i}, B_{i}$ be connected quadratic weighted graphs and $f$ be an isometry from $\prod_{i=1}^{n} A_{i}$ on $\prod_{i=1}^{m} B_{i}$. What is a sufficient condition to the following assertion?

$n=m$ and $f=\prod f_{i}$ (changing the suffix if necessary), where $f_{i}$ is an isometry from $A_{i}$ on $B_{i}$.

(ii) Let $L$ be an indecomposable positive definite quadratic lattice with $L=\tilde{L}$, and let $A$ be an associated quadratic weighted graph. If $A \cong B \times C$ where $B, C$ are quadratic weighted graphs, then is there a decomposition $L \cong M \otimes N$ so that $B$ (resp. $C$ ) is a quadratic weighted graph associated to $M$ (resp. $N)$ ?

Remark 1. For $M \cong\left(\begin{array}{lll}4 & 1 & 1 \\ 1 & 4 & 1 \\ 1 & 1 & 4\end{array}\right), N \cong\left(\begin{array}{rrr}4 & 1 & 1 \\ 1 & 4 & -1 \\ 1 & -1 & 4\end{array}\right)$, associated quadratic graphs are isometric but $M, N$ are not isometric.

Remark 2. Let $L$ be a positive definite quadratic lattice with $L$ $=\tilde{L}, m(L)=1$, and assume that $\mathfrak{M}(L) / \pm 1=A \times B$ where $A, B$ are weighted graphs with $\# A, \# B>1$. Put $\mathfrak{M}(L) / \pm 1=\left\{e_{i}\right\}$ and $e_{i}=\left(a_{i}, b_{i}\right)$ $\left(a_{i} \in A, b_{i} \in B\right)$. Suppose that there is a mapping $s_{1}$ (resp. $s_{2}$ ) from $A \times A$ (resp. $B \times B$ ) into $\{ \pm 1\}$ so that $s_{1}(a, a)=s_{2}(b, b)=1$ for every $a$ in $A$ and every $b$ in $B$, and $B\left(e_{i}, e_{j}\right)=s_{1}\left(a_{i}, a_{j}\right) s_{2}\left(b_{i}, b_{j}\right)\left[a_{i}, a_{j}\right]\left[b_{i}, b_{j}\right]$ for any $i, j$. Then we can show that there are positive definite quadratic lattices $M, N$ such that $L \cong M \otimes N, M=\tilde{M}, N=\tilde{N}, m(M)=m(N)$ $=1$ and $A, B$ are quadratic graphs associated to $M, N$ respectively. The assumption on $s_{1}, s_{2}$ is not satisfied for a decomposable lattice $M \perp N$ in Remark 1.

\section{REFERENCES}

[1] Y. Kitaoka, Scalar extension of quadratic lattices II, Nagoya Math. J. vol. 67 (1977), 159-164.

[2] - Tensor products of positive definite quadratic forms, Göttingen Nachr. Nr. 4 (1977).

[ 3 ] - Tensor products of positive definite quadratic forms II, to appear.

[ 4 ] O. T. O'Meara, Introduction to quadratic forms, Springer-Verlag, 1963.

Department of Mathematics

Nagoya University 